\title{
Heart Rate Variability in Children with Attention-Deficit/Hyperactivity Disorder: A Pilot Study
}

\author{
Malligurki Raghurama Rukmani ${ }^{a} \quad$ Shekhar P. Seshadri ${ }^{\mathrm{b}} \quad$ Kandavel Thennarasu $^{\mathrm{c}}$ \\ Trichur R. Raju ${ }^{a}$ Talakad N. Sathyaprabha ${ }^{a}$ \\ Departments of a Neurophysiology, ${ }^{\mathrm{b}}$ Child and Adolescent Psychiatry, and ${ }^{\mathrm{C} B i o s t a t i s t i c s, ~ N a t i o n a l ~ I n s t i t u t e ~ o f ~ M e n t a l ~}$ \\ Health and Neuro Sciences (NIMHANS), Bangalore, India
}

\section{Key Words}

Prefrontal cortex $\cdot$ Smart vagus $\cdot$ Heart rate variability .

Sympathovagal balance - Autonomic dysfunction .

Attention-deficit/hyperactivity disorder

\begin{abstract}
Background: Attention deficit/hyperactivity disorder (ADHD) is a common childhood neuropsychiatric disorder. Autonomic nervous system plays a vital role in attention, self-regulation, emotional stability and social affiliation, which are affected in ADHD. The prefrontal cortex, which is vital for attention, motor control, emotional regulation and higher order autonomic control, is hypofunctional in ADHD. In addition, catecholamine dysregulation is there. Purpose: We hypothesized that there is autonomic dysfunction: reduction in overall heart rate variability (HRV) and sympathovagal imbalance in children with ADHD. Methods: Study criteria were drug-naïve ADHD children who were 7-12 years of age of either gender who fulfilled DSM-IV criteria for ADHD and did not have any associated comorbid psychiat$\mathrm{ric} /$ neurological/medical disorders. Two hundred and seventy ADHD children were screened out of which only 12 were found eligible and 10 participated. Sample size was 20 (cases $=10$, age- and gender-matched healthy controls $=10$ ). Short-term HRV of both time and frequency domains were
\end{abstract}

\section{KARGER}

E-Mail karger@karger.com

www.karger.com/aon assessed by recording lead II electrocardiogram after using Tell-Show-Do, a behavior shaping technique. Comparison between groups was done using Mann-Whitney and Wilcoxon test. Demographic variables like age, height, weight and body mass index were similar between groups. Results: Among time domain parameters, SD of all NN intervals, square root of the mean of the sum of squares of differences between adjacent NN intervals and percentage of count of number of pairs of adjacent NN intervals differing by more than 50 ms were reduced in ADHD group with $p<0.05$. Among frequency domain parameters, total power was reduced in ADHD group with $p<0.05$, high frequency power (HF) was reduced in ADHD group with $p<0.01$ and low frequency power to HF ratio was higher in ADHD group with $p<0.01$. Conclusion: There is autonomic dysfunction in children with ADHD - reduction in overall HRV with sympathovagal imbalance with sympathetic dominance.

ㄷ) 2016 S. Karger AG, Basel

\section{Introduction}

Attention deficit/hyperactivity disorder (ADHD) is a common childhood neuropsychiatric disorder. It can affect the performance of child at school, behavioral interaction with others and overall well-being of the child [1].
(C) 2016 S. Karger AG, Basel

0972-7531/16/0232-0081\$39.50/0 
The constellation of deficits seen in ADHD includes inattention, hyperactivity, impulsivity, behavioral disinhibition and deficient self-regulation of emotions [2].

Various neuropsychological and neuroimaging studies have shown that there is a hypofunctional prefrontal cortex in ADHD, which is the neurobiological basis for the various symptoms seen in these individuals [3-5]. The dorsal and lateral portions of the prefrontal cortex regulate attention and motor responses while the ventral and medial portions of the prefrontal cortex regulate emotions, and the right inferior prefrontal cortex is specialized for behavioral inhibition [6]. Medial prefrontal cortex along with insular cortex also plays a vital role in higher order autonomic control $[7,8]$. Inattention, hyperactivity, emotional dysregulation and behavioral disinhibition seen in children with ADHD may be due to the hypofunctioning of prefrontal cortex [3-5]. There is a possibility that higher order autonomic control by prefrontal cortex may also be impaired in ADHD affected individuals.

Dopamine and norepinephrine (NE) are dysregulated in ADHD and are the basis for pharmacotherapy [9]. NE is an important neurotransmitter in autonomic nervous system (ANS) for it acts as a postganglionic sympathetic neurotransmitter and also is responsible for the crosstalk between various components of central autonomic network [7]. Dysregulation of NE seen in ADHD may increase the possibility of autonomic dysfunction.

Porges [10-12] proposed the polyvagal theory relating autonomic function to behavior. It specifies 2 distinct branches of vagus nerve: vegetative vagus and smart vagus. Vegetative vagus plays a vital role in the primary survival strategy of primitive vertebrates, amphibians and reptiles, which freeze when threatened. In contrast, smart vagus is mammalian and modulates sympathetic response in the service of social affiliative behavior and emotional regulation. The smart vagus inhibits acceleratory sympathetic input to the heart when sustained attention and or social engagement are adaptive and it withdraws inhibitory influence on sympathetic nervous system when situation demands fight or flee response. This theory implies that functional deficiency of the smart vagus increases the risk of emotional lability, a hallmark of psychopathology. Attention and emotional regulation, which are one of the functions of smart vagus are impaired in ADHD. This implies that there may be a possibility of functional deficiency of vagus nerve in ADHD.

Hypofunctioning of prefrontal cortex and NE dysregulation seen in ADHD and a probability of functional deficiency of smart vagus implies that there is a possibility of autonomic dysfunction. Studying the nature of cardiac autonomic dysfunction present in ADHD, if any, facilitates better understanding of the neurobiology of ADHD and assessment as to whether these children are at an increased cardiovascular risk. These may aid in treatment planning, follow-up care and improving the quality of life of children with ADHD.

Heart rate variability (HRV), the beat to beat variation of heart rate, is the result of the dynamic interplay of the sympathetic and parasympathetic inputs to the sinoatrial node $[13,14]$. It is an important non-invasive tool to study cardiac ANS $[13,15,16]$. Higher HRV reflects good adaptability to environmental and physiological needs while lower HRV indicates possible cardiovascular risk. Sympathovagal imbalance characterized by reduced vagal activity or increased sympathetic activity signifies cardiovascular morbidity [2].

There is evidence as illustrated by HRV that the sympathovagal balance is affected in various psychiatric disorders $[17,18]$. Some studies have assessed HRV in ADHD and have shown that there is autonomic dysregulation. Studies carried out by Shibagaki and Furuya [19], Beauchaine et al. [20], Buchhorn et al. [21, 22] and Musser et al. [23] showed decreased vagal tone in ADHD. However, studies carried out by Börger et al. [24], Crowell et al. [25], Negrao et al. [26] and Wang et al. [27] show that there is parasympathetic overarousal in ADHD. In addition, a study carried out by Lackschewitz et al. [28] in ADHD showed no difference in sympathovagal balance.

In a systematic review, Rash and Aguirre-Camacho [2] opined that cardiovagal control was lower in ADHD but no conclusions can be drawn because there are limited studies and results are not unanimous. Hence, the present study was aimed at assessing HRV in children with ADHD. We hypothesized that there is autonomic dysfunction - a reduction in overall HRV and sympathovagal imbalance in children with ADHD and tested it.

\section{Methods}

The study was carried out for 4 months from October 2013 to January 2014 after obtaining the requisite approval from the Institute Human Ethics Committee, National Institute of Mental Health and Neuro Sciences (NIMHANS), Bangalore, India, vide letter no. NIMH/DO/SUB-COMMITTEE/2013 (October 5, 2013). The details of the ethical clearance are as follows - 'NIMHANS IEC no: Sl. No. 2, Basic Science, MPhil. Protocol titled: Heart Rate Variability in Children with Attention-Deficit/Hyperactivity Disorder, date of Ethics Committee meeting held: August 28, 2013'. It was a prospective 2 group comparative study. Two hundred and seventy pediatric cases of $\mathrm{ADHD}$ were screened by the child and adolescent psy-
Rukmani/Seshadri/Thennarasu/Raju/ Sathyaprabha 
chiatry team in the outpatient department, NIMHANS, during the study period. Out of 270 ADHD cases, 12 cases fulfilled study criteria and out of 12 , only 10 cases participated in the present study. Equal numbers of age- and gender-matched healthy children were recruited as controls. Sample size was 20 (cases $=10$, age- and gender-matched healthy controls $=10$ ) based on availability sampling. Inclusion criteria were children fulfilling DSM-IV criteria for ADHD, age between 7 and 12 years of either gender and drug naïve. Exclusion criteria was any associated comorbid psychiatric/neurological/medical disorders as per the clinical assessment by child and adolescent psychiatrist. Our study criteria were stringent because we wanted to see the direct relation between ADHD and ANS and minimize the confounding factors. Only drug-naïve ADHD cases were included in the study because the medications prescribed for ADHD are sympathomimmetics and may influence the autonomic measures. Methylphenidate is a NE re-uptake inhibitor; guanfacine is a selective alpha2A adrenergic receptor agonist [9] and clonidine is a nonspecific alpha-2 presynaptic agonist [3]. We excluded children who had comorbid neurological, psychiatric and medical disorders because many of these disorders are associated with autonomic dysfunction. Various psychiatric disorders such as anxiety disorders, depression and schizophrenia are associated with reduction in overall HRV with sympathovagal imbalance with sympathetic dominance $[18,29]$. One of the most common pediatric neurological disorder epilepsy is associated with cardiac autonomic dysfunction [30]. Neurodevelopmental disorders such as familial dysautonomia, Allgroove syndrome, Long QT syndrome, autism, Fragile X syndrome and Rett syndrome; mitochondrial myopathies and metabolic disorders such as type 1 diabetes mellitus, Addison's disease and thyroid disorders seen in the pediatric population are also associated with autonomic dysfunction [31].

The parents of the research subjects were explained about the nature of the study and an informed written consent was obtained. A detailed history, clinical examination and anthropometric measurements were done for the recruited subjects. Tell-Show-Do, a technique of behavior shaping, was employed to establish communication with the child and perform the test. This involved explaining the procedure in a known language using phrases appropriate to the developmental level of the child (tell), demonstration of the procedure (placing 3 electrocardiography [ECG] electrodes on the thorax of the child's parent or guardian for a few minutes) (show) and, then, performing the actual procedure on the child (do) [32].

According to a study by Kim et al. [33], heart rate and ratio of low frequency power to high frequency power (LF/HF) ratio showed a diurnal pattern, with a minimum value during nocturnal sleep, a sharp increase upon waking in the morning and a maximum value in the afternoon (working time). Hence, the ECG recording was done for all the subjects in the present study between 9.00 a.m. and 11.00 a.m. in the morning to ensure that diurnal variation in HRV is similar between all the study subjects. Resting lead II ECG was recorded for $15 \mathrm{~min}$ in the autonomic laboratory, Department of Neurophysiology, NIMHANS. The subjects were allowed to rest in the supine position for about $15 \mathrm{~min}$ to obtain steady-state hemodynamics before the commencement of the ECG recording. The lab has an analogue digital converter (Power Lab, 16 channels data acquisition system, AD Instruments, Australia) with a sampling rate of $1,024 \mathrm{~Hz}$, through which the ECG signals are conveyed. The data acquired were stored in a personal computer and analyzed offline with the help of HRV Analysis Software version 1.1 (Power Lab AD Instruments, Australia).
An ectopic free 5 min segment was selected from the $15 \mathrm{~min}$ ECG recording and analyzed to get time and frequency domain parameters (linear methods) of the short-term HRV according to the standards established by the Task Force of the European Society of Cardiology and The North American Society of Pacing and Electrophysiology (1996).

\section{Time Domain Measurement of Short-Term HRV:}

With time domain measurement of HRV, either the heart rate at any point of time or the intervals between successive normal complexes are determined. In the ECG, each QRS complex is detected, and then, all the intervals between adjacent QRS complexes (NN/normal to normal intervals) resulting from sinus node depolarization and instantaneous heart rate are determined. The components of time domain measurement of short-term HRV are heart rate, mean of all normal to normal intervals, SD of all $\mathrm{NN}$ intervals (SDNN), square root of the mean of the sum of squares of differences between adjacent NN intervals (RMSSD), count of number of pairs of adjacent NN intervals differing by more than $50 \mathrm{~ms}$ (NN50) and percentage of NN50 count of all $\mathrm{NN}$ intervals (pNN50). SDNN is sensitive to all sources of heart rate variation and represents overall HRV while RMSSD, NN50 and pNN50 are most sensitive to parasympathetic activity/vagal tone $[13,16]$.

\section{Frequency Domain Measurement of Short-Term HRV}

In order to get deeper insight into the dynamics and components of HRV, frequency domain of HRV was measured using a more advanced second order statistics known as power spectral density (PSD) analysis. With PSD analysis, heart rate signal is decomposed into frequency components and quantified in terms of their relative intensity termed as 'power'. PSD analysis is one of the spectral methods, which provides the basic information as to how overall HRV, the power (i.e., variance of RR intervals or heart rate), is distributed as a function of frequency into different components. Fast Fourier transformation was then employed to provide frequency-specific information of heart rate behavior. The components of frequency domain of short-term HRV are total power (TP), sum of the constituent frequencies; HF, the power of frequency band ranging from 0.15 to $0.4 \mathrm{~Hz}$; LF, the power of frequency band ranging from 0.04 to $0.15 \mathrm{~Hz}$; $\mathrm{HF}$ in normalized units (HF nu); LF in LF normalized units (LF nu); and LF/HF ratio, the ratio of the power of LF component to the power of HF component. TP is sensitive to all sources of heart rate variation reflecting overall HRV. HF is sensitive to parasympathetic/vagal tone while LF is sensitive to both sympathetic and parasympathetic tone but predominantly sympathetic tone for practical purposes. LF/HF ratio signifies sympathovagal balance. An increase in LF/HF ratio signifies sympathetic dominance while a reduction in $\mathrm{LF} / \mathrm{HF}$ ratio signifies parasympathetic dominance $[13,16]$.

\section{Statistical Analysis}

Frequency domain and time domain parameters of short-term HRV were tested for normal distribution. The data did not follow normal distribution, and hence, the comparison between ADHD subjects and healthy controls was done using non-parametric Mann-Whitney and Wilcoxon test. Spearman rank correlation coefficient was used to calculate the correlation between shortterm HRV and confounding variables such as age, height, weight and body mass index (BMI). 
Table 1. Demographic details of the study subjects

\begin{tabular}{|c|c|c|c|c|c|c|}
\hline Variables & ADHD group & Control group & Mann-Whitney U & Wilcoxon W & $Z$ value & $\mathrm{p}$ value \\
\hline Age, years & 8.00 & 8.00 & 50.00 & 105.00 & 0.000 & 1.000 \\
\hline Height, $\mathrm{cm}$ & 129.50 & 135.00 & 41.00 & 96.00 & -0.681 & 0.496 \\
\hline $\mathrm{BMI}, \mathrm{kg} / \mathrm{m}^{2}$ & 16.23 & 14.80 & 36.50 & 91.50 & -1.021 & 0.307 \\
\hline
\end{tabular}

Values expressed as median.

Table 2. Comparison of time domain measures of short term HRV of ADHD children and healthy controls

\begin{tabular}{|c|c|c|c|c|c|c|}
\hline HRV variables & ADHD group & Control group & Mann-Whitney U & Wilcoxon W & $Z$ value & $\mathrm{p}$ value \\
\hline Heart rate in beats/min & 87.00 & 79.00 & 30.50 & 85.50 & -1.481 & 0.139 \\
\hline Mean NN, ms & 684.76 & 758.87 & 30.00 & 85.00 & -1.512 & 0.131 \\
\hline RMSSD, ms & 36.50 & 55.03 & 19.00 & 74.00 & -2.343 & $0.019^{*}$ \\
\hline NN50, count & 64.00 & 134.00 & 25.00 & 80.00 & -1.891 & 0.059 \\
\hline pNN50, \% & 16.62 & 36.09 & 23.00 & 78.00 & -2.041 & $0.041^{*}$ \\
\hline
\end{tabular}

Values expressed as median, ${ }^{*} \mathrm{p}<0.05$.

Mean NN $=$ Mean of all normal to normal intervals.

Table 3. Comparison of frequency domain measures of short-term HRV of ADHD children and healthy controls

\begin{tabular}{|c|c|c|c|c|c|c|}
\hline HRV variables & ADHD group & Control group & Mann-Whitney U & Wilcoxon W & $Z$ value & $\mathrm{p}$ value \\
\hline $\mathrm{TP}, \mathrm{m}^{2}$ & $1,428.80$ & $2,515.45$ & 21.00 & 76.00 & -2.192 & $0.028^{*}$ \\
\hline $\mathrm{LF}, \mathrm{m}^{2}$ & 320.72 & 531.11 & 25.00 & 80.00 & -1.890 & 0.059 \\
\hline $\mathrm{LF}, \mathrm{nu}$ & 45.51 & 32.39 & 26.00 & 81.00 & -1.814 & 0.070 \\
\hline $\mathrm{HF}, \mathrm{nu}$ & 26.70 & 50.94 & 28.00 & 83.00 & -1.663 & 0.096 \\
\hline LF/HF ratio & 1.84 & 0.71 & 11.00 & 66.00 & -2.950 & $0.003^{* *}$ \\
\hline
\end{tabular}

Values expressed as median. ${ }^{*} \mathrm{p}<0.05,{ }^{* *} \mathrm{p}<0.01$.

$\mathrm{LF} / \mathrm{HF}$ ratio $=$ Sympathovagal balance.

\section{Results}

\section{Clinical Profile and Demographic Details}

Among the 20 study subjects, 10 were children with ADHD (ADHD group) and other 10 were age- and gendermatched healthy controls (control group). There were 8 males and 2 females in either group. In ADHD group, 9 had ADHD combined type and 1 had ADHD predominantly inattention type. The demographic variables like age, height, weight and BMI were statistically similar between ADHD group and control group and are represented in table 1 .

\section{Time Domain Measures}

Time domain measures of short-term HRV are represented in table 2 . SDNN was statistically significantly reduced in ADHD group with $\mathrm{p}=0.017$. RMSSD was statistically significantly reduced in ADHD group with $\mathrm{p}=$ 0.019 . In addition, pNN50 was statistically significantly reduced in ADHD group with $\mathrm{p}=0.041$.

\section{Frequency Domain Measures}

Frequency domain measures of short-term HRV are represented in table 3. TP was statistically significantly reduced in ADHD group with $\mathrm{p}=0.028$. LF was reduced
84

Ann Neurosci 2016;23:81-88 DOI: $10.1159 / 000443574$
Rukmani/Seshadri/Thennarasu/Raju/ Sathyaprabha 
Table 4. Correlation of time domain measures of HRV with age, height, weight and BMI

\begin{tabular}{|c|c|c|c|c|c|}
\hline HRV variables & Spearman's rho & Age, years & Height, $\mathrm{cm}$ & Weight, kg & BMI, $\mathrm{kg} / \mathrm{m}^{2}$ \\
\hline Heart rate, beats/min & $\begin{array}{l}\text { Correlation coefficient } \\
\text { p value }\end{array}$ & $\begin{array}{r}-0.091 \\
0.701\end{array}$ & $\begin{array}{r}-0.238 \\
0.313\end{array}$ & $\begin{array}{r}-0.085 \\
0.722\end{array}$ & $\begin{array}{l}0.253 \\
0.281\end{array}$ \\
\hline Mean NN, m & $\begin{array}{l}\text { Correlation coefficient } \\
\text { p value }\end{array}$ & $\begin{array}{l}0.074 \\
0.755\end{array}$ & $\begin{array}{l}0.231 \\
0.327\end{array}$ & $\begin{array}{l}0.060 \\
0.803\end{array}$ & $\begin{array}{r}-0.256 \\
0.275\end{array}$ \\
\hline RMSSD, m & $\begin{array}{l}\text { Correlation coefficient } \\
\text { p value }\end{array}$ & $\begin{array}{r}-0.187 \\
0.430\end{array}$ & $\begin{array}{l}0.017 \\
0.945\end{array}$ & $\begin{array}{r}-0.241 \\
0.306\end{array}$ & $\begin{array}{c}-0.456^{*} \\
0.043\end{array}$ \\
\hline NN50, count & $\begin{array}{l}\text { Correlation coefficient } \\
\text { p value }\end{array}$ & $\begin{array}{r}-0.204 \\
0.389\end{array}$ & $\begin{array}{r}-0.022 \\
0.926\end{array}$ & $\begin{array}{r}-0.243 \\
0.301\end{array}$ & $\begin{array}{r}-0.406 \\
0.076\end{array}$ \\
\hline
\end{tabular}

${ }^{*} \mathrm{p}<0.05,{ }^{* *} \mathrm{p}<0.01$

Mean $\mathrm{NN}=$ Mean of all normal to normal intervals.

in ADHD group but was not statistically significant. HF was statistically significantly reduced in ADHD group with $\mathrm{p}=0.008$. LF in normalized units was higher in ADHD group but was not statistically significant. HF in normalized units was reduced in ADHD group but was not statistically significant. LF/HF ratio was statistically significantly higher in $\mathrm{ADHD}$ group as compared to control group with $\mathrm{p}=0.003$.

\section{Non-Parametric Correlation Analysis between}

Time Domain Measures of HRV and Confounding

Variables: Age, Height, Weight and BMI

Correlation analysis between time domain measures and confounding variables like age, height, weight and BMI using Spearman's rank correlation coefficient is represented in table 4 . There was no statistically significant correlation between time domain measures of short-term HRV and age, height and weight. There was significant negative correlation between BMI and SDNN with $\mathrm{p}=$ 0.007 . There was significant negative correlation between BMI and RMSSD with $\mathrm{p}=0.043$.

\section{Non-Parametric Correlation Analysis between}

Frequency Domain Measures of HRV and

Confounding Variables: Age, Height, Weight and BMI

Correlation analysis between frequency domain measures and confounding variables like age, height, weight and BMI using Spearman's rank correlation coefficient is represented in table 5 . There was no statistically signifi- cant correlation between frequency domain measures of short-term HRV and age, height and weight. There was significant negative correlation between BMI and TP with $\mathrm{p}=0.002$. There was significant negative correlation between BMI and LF with $\mathrm{p}=0.000$ and BMI and HF with $\mathrm{p}=0.012$.

\section{Discussion}

The study results of time domain and frequency domain measures of short-term HRV in children with ADHD and age- and gender-matched healthy controls supports our hypothesis that there is autonomic dysfunction - a reduction in overall HRV with sympathovagal imbalance, in children with ADHD.

Among time domain parameters, SDNN, RMSSD and pNN50 were reduced in ADHD group with $p<0.05$. A reduction in SDNN signifies a reduction in overall HRV while a reduction in RMSSD and pNN50 signifies a reduction in parasympathetic activity [13]. Similar results were obtained in studies carried out by Buchhorn et al. $[21,22]$ in which RMSSD and pNN50 were lower in ADHD cases as compared to controls. However, groups did not differ in SDNN in that study unlike our study. The present study results are in contradiction to results of studies carried out by Lackschewitz et al. [28] and Negrao et al. [26]. In the study carried out by Lackschewitz et al. [28], SDNN and RMSSD values in adults with 
Table 5. Correlation of frequency domain measures of HRV with age, height, weight and BMI

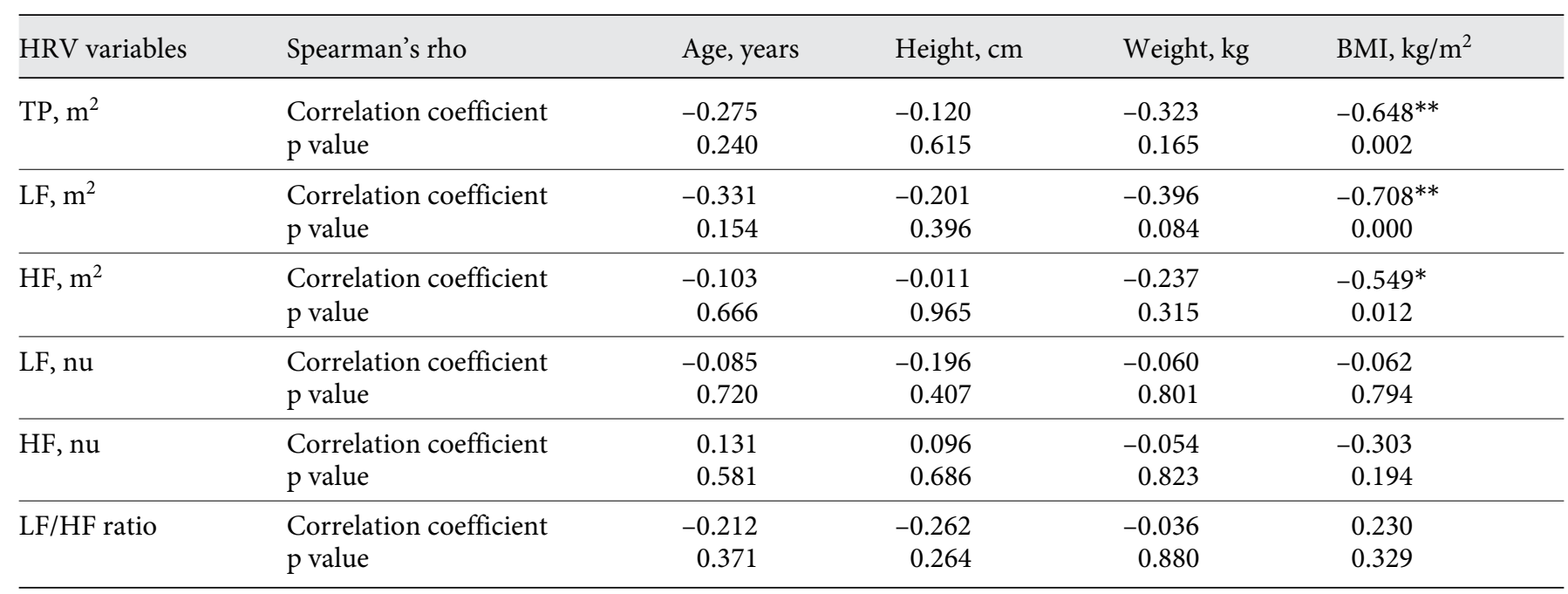

${ }^{*} \mathrm{p}<0.05,{ }^{* *} \mathrm{p}<0.01$.

$\mathrm{LF} / \mathrm{HF}$ ratio $=$ Sympathovagal balance.

ADHD were not statistically different from control group. In the study carried out by Negrao et al. [26], RMSSD was higher in ADHD children as compared to controls.

Among frequency domain parameters, TP was reduced in ADHD group with $\mathrm{p}<0.05$, HF power was reduced in ADHD group with $\mathrm{p}<0.01$ and LF/HF ratio was higher in ADHD group with $\mathrm{p}<0.01$. A reduction in TP signifies a reduction in overall $\mathrm{HRV}$. A reduction in HF power signifies reduced parasympathetic activity. An increase in LF/HF ratio signifies that there was sympathovagal imbalance with sympathetic dominance [13]. Similar results were obtained in studies carried out by Shibagaki and Furuya [19] and Beauchaine et al. [20]. In the study carried out by Shibagaki and Furuya [19], high frequency component of HRV was reduced in children with ADHD as compared to controls during baseline and listening tasks. In the study carried out by Beauchaine et al. [20], high frequency component of HRV was significantly lower in $\mathrm{ADHD} /$ conduct disorder group compared to ADHD and control group during baseline. High frequency component of HRV in children with ADHD was also lower than controls but was not statistically significant. The study results are in contradiction to results of studies carried out by Börger et al. [24], Lackschewitz et al. [28], Negrao et al. [26] and Wang et al. [27]. In the study carried out by Börger et al. [24], the high frequency component was higher in ADHD affected children as compared to healthy children. In the study carried out by
Lackschewitz et al. [28], there was no statistically significant difference in LF (nu), HF (nu) and LF/HF ratio between ADHD adults and controls. In the study carried out by Negrao et al. [26], one of the time domain measures of HRV, RMSSD, was higher in stimulant-free ADHD children as compared to controls but there was no statistically significant difference in frequency domain measures. In the study carried out by Wang et al. [27], only the preschool aged boys with inattentive and hyperactive features had decreased LF and increased HF but not the girls.

In addition, our study showed that there is statistically significant negative correlation between BMI and overall HRV (SDNN and TP), parasympathetic activity (RMSSD and HF) and sympathetic activity (LF). This is supported by a study carried out by Baek et al. [34] which shows that SDNN, RMSSD and HF are reduced in individuals with high BMI.

Evidence from neuropsychological studies and functional brain imaging studies such as single-photon emission CT, functional MRI and positron emission tomography in individuals with ADHD have shown that neural activity is reduced in prefrontal cortex $[3,6,35]$. Interestingly, this area is involved in generation of autonomic tone and plays a vital role in higher order autonomic control $[7,8]$. Noradrenaline, one of the important catecholamines, the optimal levels of which are essential for both the functioning of prefrontal cortex and cross talk between various components of central autonomic net- 
work is also dysregulated in ADHD [6-8]. Hypofunctioning of the prefrontal cortex and dysregulation of catecholamines might have been responsible for the autonomic dysfunction - reduction in overall HRV and sympathovagal imbalance, seen in children with ADHD. Reduced vagal tone observed in children with $\mathrm{ADHD}$ might be as a result of functional deficiency of smart vagus, for this plays a vital role in carrying the parasympathetic output of the central autonomic network, social affiliation, sustained attention and emotional regulation [10-12]. Thus, it is plausible that disrupted autonomic regulation seen in children with ADHD might be central in origin. Furthermore, in our study, children with ADHD did not have any comorbidity and were drug naive suggesting less likelihood of result being confounded by other variables.

Limitation of the present study was that the sample size was low. A larger sample size would have been better to validate the study results. However, it is practically challenging to get a larger sample of ADHD children who are drug naïve and do not have any comorbid disorders because a significant number of ADHD children have a high rate of comorbid psychiatric or neurological disorders such as oppositional defiant disorder, conduct disorder, anxiety disorders, learning disorders, Tourette's disorder, mood disorders or epilepsy $[3,36$, 37].

Our study helped in unraveling the nature of cardiac autonomic dysfunction present in children with ADHD. This facilitates better understanding of the neurobiology of ADHD. It also signifies that the children with ADHD are at an increased cardiovascular risk for a reduction in overall HRV, reduced vagal activity or increased sympathetic activity positively correlated with an increased incidence of cardiovascular comorbid conditions [2]. In addition, in a systematic review by Martinez-Raga et al. [38], the reviewers opine that there is a minimal risk for serious cardiovascular adverse events with stimulants prescribed for ADHD, although the benefits outweigh the risks. This suggests that children with ADHD should be followed up regularly for cardiac evaluation and great caution is preferable while considering medical line of treatment for ADHD affected individuals with personal or family history or other known risk factors for cardiovascular disease. All these may aid in treatment planning, follow-up care and improving the quality of life of children with ADHD. However, the findings of the present study cannot be generalized for it is limited by a small sample size. Further study with a larger sample size needs to be carried out to validate these findings.

\section{Acknowledgment}

We thank NIMHANS, Bangalore, India, for providing the facilities to carry out the present study. We express gratitude to Satish Chandra Girimaji, Professor and Head of the Department, Department of Child and Adolescent Psychiatry, NIMHANS, Bangalore for his valuable inputs and support to conduct the study.

\section{Author Contribution}

M.R.R. was involved in designing the study and drawing the hypothesis. She recruited research subjects for the study, recorded HRV, did the HRV analysis, interpreted the study results and wrote the manuscript under the guidance of T.N.S., S.P.S., T.R.R. and K.T.

S.P.S. gave his valuable inputs and guided M.R.R. in designing the study, interpretation of the results and writing the manuscript. He did clinical examination of the study subjects and guided M.R.R. to recruit the research subjects.

K.T. gave his valuable inputs for designing the study and writing the manuscript and for the statistical analysis.

T.R.R. gave his valuable inputs for designing the study, interpretation of the results and writing the manuscript.

T.N.S. guided M.R.R. in designing the study, recording HRV, interpretation of the results and writing the manuscript.

\section{Disclosure Statement}

Authors report no biomedical or financial interests or potential conflicts of interest.

\section{References}

1 Subcommittee on Attention-Deficit/Hyperactivity Disorder; Steering Committee on Quality Improvement and Management, Wolraich $\mathrm{M}$, Brown L, Brown RT, DuPaul G, Earls M, Feldman HM, Ganiats TG, Kaplanek B, Meyer B, Perrin J, Pierce K, Reiff M, Stein MT, Visser S: ADHD: clinical practice guideline for the diagnosis, evaluation, and treatment of attention- deficit/hyperactivity disorder in children and adolescents. Pediatrics 2011;128:1007-1022.

2 Rash JA, Aguirre-Camacho A: Attention-deficit hyperactivity disorder and cardiac vagal control: a systematic review. Atten Defic Hyperact Disord 2012;4:167-177.

3 Martin A, Volkmar FR, Lewis M: Lewis's Child and Adolescent Psychiatry: A Compre- hensive Textbook, ed 4. Philadelphia, Lippincott Williams \& Wilkins, 2007.

4 Gozal D, Molfese DL: Attention Deficit Hyperactivity Disorder: From Genes to Patients. New Jersey, Humana Press, 2005.

5 Tripp G, Wickens JR: Neurobiology of ADHD. Neuropharmacology 2009;57:579589. 
6 Arnsten AF: The emerging neurobiology of attention deficit hyperactivity disorder: the key role of the prefrontal association cortex. J Pediatr 2009;154:I-S43.

7 Benarroch EE: The central autonomic network: functional organization, dysfunction, and perspective. Mayo Clin Proc 1993;68: 988-1001.

8 Low PA, Benarroch EE: Clinical Autonomic Disorders, ed 3. Philadelphia, Lippincott Williams \& Wilkins, 2008.

9 Curatolo P, D’Agati E, Moavero R: The neurobiological basis of ADHD. Ital J Pediatr 2010;36:79.

10 Beauchaine TP, Gatzke-Kopp L, Mead HK: Polyvagal theory and developmental psychopathology: emotion dysregulation and conduct problems from preschool to adolescence. Biol Psychol 2007;74:174-184.

11 Porges SW: The polyvagal perspective. Biol Psychol 2007;74:116-143.

12 Porges SW: The polyvagal theory: new insights into adaptive reactions of the autonomic nervous system. Cleve Clin J Med 2009; 76(suppl 2):S86-S90.

13 Malik M: Clinical Guide to Cardiac Autonomic Tests. Dordrecht, Kluwer Academic Publishers, 1998.

14 Yamada T: Tachycardia. New York, Intech, 2012.

$15 \mathrm{Kemp}$ AH, Quintana DS: The relationship between mental and physical health: insights from the study of heart rate variability. Int J Psychophysiol 2013;89:288-296.

16 Heart rate variability. Standards of measurement, physiological interpretation, and clinical use. Task force of the European society of cardiology and the North American society of pacing and electrophysiology. Eur Heart J 1996; 17:354-381.

17 Malik M, Cramm AJ: Dynamic Electrocardiography. New York, Futura, 2004.
18 Udupa K, Sathyaprabha TN, Thirthalli J, et al: Alteration of cardiac autonomic functions in patients with major depression: a study using heart rate variability measures. J Affect Disord 2007;100:137-141.

19 Shibagaki M, Furuya T: Baseline respiratory sinus arrhythmia and heart-rate responses during auditory stimulation of children with attention-deficit hyperactivity disorder. Percept Mot Skills 1997;84(3 pt 1):967-975.

20 Beauchaine TP, Katkin ES, Strassberg Z, et al: Disinhibitory psychopathology in male adolescents: discriminating conduct disorder from attention-deficit/hyperactivity disorder through concurrent assessment of multiple autonomic states. J Abnorm Psychol 2001; 110:610-624

21 Buchhorn R, Conzelmann A, Willaschek C, et al: Heart rate variability and methylphenidate in children with ADHD. Atten Defic Hyperact Disord 2012;4:85-91.

22 Buchhorn R, Müller C, Willaschek C, et al: How to predict the impact of methylphenidate on cardiovascular risk in children with attention deficit disorder: methylphenidate improves autonomic dysfunction in children with ADHD. ISRN Pharmacol 2012;2012: 170935.

23 Musser ED, Backs RW, Schmitt CF: Emotion regulation via the autonomic nervous system in children with attention-deficit/hyperactivity disorder (ADHD). J Abnorm Child Psychol 2011;39:841-852.

24 Börger N, van der Meere J, Ronner A, et al: Heart rate variability and sustained attention in ADHD children. J Abnorm Child Psychol 1999;27:25-33.

25 Crowell SE, Beauchaine TP, Gatzke-Kopp L, et al: Autonomic correlates of attention-deficit/hyperactivity disorder and oppositional defiant disorder in preschool children. J Abnorm Psychol 2006;115:174-178.

26 Negrao BL, Bipath P, van der Westhuizen D, et al: Autonomic correlates at rest and during evoked attention in children with attentiondeficit/hyperactivity disorder and effects of methylphenidate. Neuropsychobiology 2011; 63:82-91.
27 Wang TS, Huang WL, Kuo TB, et al: Inattentive and hyperactive preschool-age boys have lower sympathetic and higher parasympathetic activity. J Physiol Sci 2013;63:87-94.

28 Lackschewitz H, Hüther G, Kroner-Herwig B: Physiological and psychological stress responses in adults with attention-deficit/hyperactivity disorder (ADHD). Psychoneuroendocrinology 2008;33:612-624.

29 Yang AC, Hong CJ, Tsai SJ: Heart rate variability in psychiatric disorders. Taiwanese J Psychiatr (Taipei) 2010;24:99-109.

30 Jansen K, Lagae L: Cardiac changes in epilepsy. Seizure 2010;19:455-460.

31 Axelrod F, Chelimsky GG, Weese-Mayer DE: Pediatric autonomic disorders. Pediatrics 2007;118:309-321.

32 American Academy of Pediatric Dentistry: Guideline on Behavior Guidance for the Pediatric Dental Patient. Reference Manual, 2011, vol 34, pp 170-182.

33 Kim HS, Yoon KH, Cho JH: Diurnal heart rate variability fluctuations in normal volunteers. J Diabetes Sci Technol 2014;8:431-433.

34 Baek J, Park D, Kim I, et al: Autonomic dysfunction of overweight combined with low muscle mass. Clin Auton Res 2013;23:325331.

35 McCarthy H, Skokauskas N, Frodl T: Identifying a consistent pattern of neural function in attention deficit hyperactivity disorder: a meta-analysis. Psychol Med 2014;44:869880

36 Coffey CW, Brumback RA: Pediatric Neuropsychiatry. Philadelphia, Lippincott Williams \& Wilkins, 2006.

37 Tasman A, Kay J, Lieberman JA: Psychiatry, ed 2. Chichester, John Wiley \& Sons, 2003.

38 Martinez-Raga J, Knecht C, Szerman N, et al: Risk of serious cardiovascular problems with medications for attention-deficit hyperactivity disorder. CNS Drugs 2013;27:15-30. 